\title{
Peripheral cell line suppression in dengue infection in relation to serological status
}

\author{
Vani Krishnamurthy', Rajalakshmi Rajashekar ${ }^{2}$, Srinivasa Murthy Doreswamy ${ }^{3}$ \\ ${ }^{1,2}$ Assistant Professor, Department of Pathology, ${ }^{3}$ Professor, Department of Pediatrics, JSS Medical College, \\ JSS University, Mysuru, Karnataka, India
}

Background: Dengue infection occurs in epidemic and can be fatal. Bleeding due to thrombocytopenia and hypovolemic shock due to capillary leak is the predominant clinical feature. Bone marrow suppression as a cause of thrombocytopenia and neutropenia is well documented. This is generally recognised as decrease in the count of formed elements in the peripheral blood. However, relation of such a phenomenon with the serological stage of the disease is less studied. Aims and Objective: We aimed to quantify the magnitude of peripheral cell line suppression in relation to serological status of dengue illness. Materials and Methods: This was a retrospective study. Dengue infection was diagnosed by clinical and serological parameters. The proportion of patients with peripheral cell line suppression and their association with the different serological phases was described. Results: Records of 219 Dengue positive patients were analysed retrospectively. Most common serological status was positive lgG which was noted in $68.9 \%$, this was followed by NS1 antigen positivity in $39.7 \%$ of the patients. Around $40 \%$ of the patients had peripheral cell line suppression of two or more formed elements at the time of diagnosis. Sixty-four percent of them had low platelet and WBC counts. All the three formed elements were decreased in $14 \%$ of our patients. Of these, $23 \%$ were in the initial phase of the illness and $33.3 \%$ during post primary phase. Conclusion: Decrease in the formed elements in the peripheral blood suggesting bone marrow suppression is seen in nearly $40 \%$ of the patients with Dengue infection at the time of diagnosis. This was highest during post primary phase followed by initial phase of the illness.

Access this article online

Website:

http://nepjol.info/index.php/AJMS

DOI: 10.3126/ajms.v7i5.15012

E-ISSN: 2091-0576

P-ISSN: 2467-9100

Key words: Thrombocytopenia, Neutropenia, Dengue infection, Bone marrow suppression

\section{INTRODUCTION}

Dengue is a regularly occurring epidemic in India. Many of the patients have non-fatal illness and recover with minimal medical intervention. The exact hospitalisation rates are not known. However, WHO states that, nearly 50000 hospitalisations occur worldwide for severe Dengue infection. Mortality in well-treated Dengue infection is reported to be less than $1 \%{ }^{1}$ The two predominant pathogenic mechanisms that can cause death in Dengue infection is bleeding due to thrombocytopenia and hypovolemic shock due to capillary leak. Animal experiments have demonstrated that the thrombocytopenia in Dengue is predominantly due to decreased production of megakaryocytes. ${ }^{2}$
However, current evidences suggest that complex immune mechanisms play a significant role in causing thrombocytopenia. ${ }^{3-5}$

Many of the patients with thrombocytopenia also demonstrate reduction in the counts of other cell lines indicating hypo functioning bone marrow. ${ }^{6}$ Such a phenomenon is now increasingly being recognised. The extent and pattern of bone marrow suppression as inferred by decrease in different cell lines in the peripheral blood in Dengue infected patients and its relation to different serological phase of the illness is yet to be characterised. This information could be of significant value as it enables clinicians inmanagement of cases. This study intended to provide information on the magnitude and pattern of cell 
line suppression, and relation to the different phases of Dengue infection.

\section{Primary objective}

To describe the magnitude of formed element suppression in the peripheral blood in relation to serological stage of Dengue infection.

\section{Secondary objective}

To describe the pattern of different cell lines affected in patients diagnosed with Dengue infection.

\section{MATERIALS AND METHODS}

\section{Study design}

This is a retrospective descriptive study conducted in a tertiary care centre. Study period was from January 2014 to December 2014.

\section{Inclusion criteria}

Patients aged more than one year admitted with fever and had serological diagnosis of Dengue infection.

\section{Exclusion criteria}

Patients who were diagnosed as Dengue elsewhere and admitted to the hospital for further management.

\section{Subjects and investigations}

All the patients who full filled the inclusion criteria were included in the analysis. Patients who were admitted with fever and had at least one serological evidence of Dengue infection such as positive NS1 antigen or Dengue IgM or $\operatorname{IgG}$ antibody were considered to be suffering from Dengue infection.

\section{Serological investigations}

Serological investigations were done in the department of microbiology on clotted blood samples. NS1 antigen, $\operatorname{IgM}$ and $\operatorname{IgG}$ antibodies were detected by Immunochromatography technique (Standard Diagnostic Inc. Bioline, Dengue duo, Gyeibggu-do, Republic of Korea). The results were reported as positive or negative.

\section{Haematological investigations}

All the haematological investigations were done on EDTA samples. The analysis was done by the automated cell counter SysmexXN- 1000(Sysmex America, Inc. in Lincolnshire, Illinois). Haemoglobin was expressed as $\mathrm{Gram} / \mathrm{dl}, \mathrm{RBC}, \mathrm{WBC}$ and platelet counts were expressed as cells $/ \mathrm{mm} 3$. Bone marrow suppression was presumed as the cause of decrease in two or more formed elements in the peripheral blood. The RBC count of 4 million $/ \mathrm{mm}^{3}$, WBC count of $4000 / \mathrm{mm}^{3}$ and platelet count of $150000 / \mathrm{mm}^{3}$ were considered to be the lowest values below which they were considered to be reduced.

\section{Data collection}

The medical record numbers of all the patients diagnosed as Dengue between Jan 2014 and Dec 2014 (both inclusive), were obtained from medical records department. These numbers were individually searched in the repository of laboratory information system of our hospital. The results of serological investigations were noted. Later the haematological values of the sample sent at the time of diagnosis were noted. Subsequent haematological values were not obtained for this study. These values were entered in Microsoft excel and later analysed.

\section{Ethical consideration}

This study was approved by the institutional ethical committee with exemption for patient consent.

\section{Sample size}

Our initial observation revealed that bone marrow suppression in Dengue infection ranged from 25 to 30\%. Assuming arate of $25 \%$ with the margin of error $5 \%$ and confidence limits of $90 \%$, we needed 201 cases for this study.

\section{Statistical analysis}

The continuous variables with normal distribution were reported as mean and standard deviation. Continuous variables with skewed distribution were reported as median and inter quartile range. Categorical variables were reported as proportions. All the analysis was done using Microsoft Excel 2013.

\section{RESULTS}

We had a total of 368 patients diagnosed and treated for Dengue infection in our hospital during the study period. Out of them, 144 patients had the diagnosis done elsewhere and were admitted to our hospital for management alone. We could not get complete data needed in five patients. We had 219 subjects for final analysis.

The median age of patients was 23 years and $56 \%$ were males. The base line characteristics of our study subjects are depicted in Table 1. We had 41 children aged less than 12 years which constituted $18.7 \%$. Our youngest patient was 1 year and oldest was 79 years. Lowest haemoglobin noted in our study was $6.2 \mathrm{gm} / \mathrm{dl}$ and highest was $21.5 \mathrm{gm} / \mathrm{dl}$. Lowest WBC count noted was $500 / \mathrm{mm}^{3}$ and highest was $18500 / \mathrm{mm}^{3}$. Lowest platelet count was $5000 / \mathrm{mm}^{3}$ and highest was $394000 / \mathrm{mm}^{3}$.

$68.9 \%$ of our patients had $\operatorname{IgG}$ antibody positive at diagnosis followed by NS1 antigen in 39.7\%. Detailed 
serological profile of our study subjects is depicted in Table 2.

Haematological profile: Twenty $(9.1 \%)$ of our study subjects had Haemoglobin of less than $10 \mathrm{gm} / \mathrm{dl}$, whereas $72(32.9 \%)$ had haemoglobin of greater than $14 \mathrm{gm} / \mathrm{dl}$. Fifty-nine $(26.9 \%)$ of the patients had low haematocrit of less than $35 \%$ and $32(14.6 \%)$ had a haematocrit greater than $45 \%$. Seventy-two $(32.9 \%)$ had WBC count below $4000 / \mathrm{mm}^{3}$ and $18(8.2 \%)$ had the counts above $11000 / \mathrm{mm}^{3}$. One hundred and seventy-six $(80.4 \%)$ had thrombocytopenia with platelet count of less than $150000 / \mathrm{mm}^{3}$ and $69(31.5 \%)$ patients had a platelet count of less than $50000 / \mathrm{mm}^{3}$.

Bone marrow suppression as inferred by decrease in two or more cell lines in the peripheral blood was seen in $87(39.7 \%)$ of our study subjects. Most of them had decreased platelet and WBC counts. All the three cell lines were decreased in $12(5 \%)$ of our subjects. Table 3 depicts the peripheral cell line suppression profile in our study subjects. Twenty-nine $(33.3 \%)$ of the patients with two or more cell line suppression had only $\operatorname{IgG}$ positive. This was followed by $20(23 \%)$ who had only NS1 antigen positive. Figure 1 depicts the proportion of patients with peripheral cell line suppression associated different serological phase of Dengue infection.

\begin{tabular}{lc}
\multicolumn{2}{l}{ Table 1: Baseline characteristics } \\
\hline Total (N) & $\mathbf{2 1 9}$ \\
\hline Age (yrs) - median (IQR) & $23(14-35.5)$ \\
Gender Male - $\mathrm{n}(\%)$ & $123(56)$ \\
Gender Female - $\mathrm{n}(\%)$ & $96(44)$ \\
Hb (gm/dl) Mean (SD) & $13.1(2.5)$ \\
WBC/cumm - Median (IQR) & $5075(3315-7953)$ \\
Platelet count - Median (IQR) & $80000(36000-130000)$ \\
\hline
\end{tabular}

\section{Table 2: Serological profile in dengue illness}

\begin{tabular}{lc}
\hline Positive serological parameter at diagnosis & Number (\%) \\
\hline NS1 Antigen & $87(39.7)$ \\
IgM antibody & $54(24.7)$ \\
IgG antibody & $151(68.9)$ \\
NS1 Antigen \& IgM antibody & $18(8.2)$ \\
NS1 Antigen \& IgG antibody & $34(15.5)$ \\
IgM antibody \& IgG antibody & $28(12.8)$ \\
\hline
\end{tabular}

\begin{tabular}{lc}
$\begin{array}{l}\text { Table 3: Peripheral cell line suppression profile } \\
\text { in dengue illness }\end{array}$ \\
\hline $\begin{array}{l}\text { Decreased cell lines in } \\
\text { the peripheral blood }\end{array}$ & $\begin{array}{c}\text { Number }(\% \text { of } \\
\text { total cases) }\end{array}$ \\
\hline Platelet \& WBC & $56(26)$ \\
Platelet \& RBC & $19(9)$ \\
RBC \& WBC & $0(0)$ \\
Platelet, WBC \& RBC & $12(5)$ \\
Total & $87(39.7)$ \\
\hline
\end{tabular}

\section{DISCUSSION}

Bone marrow involvement in Dengue has been a recognised phenomenon and several animal and human studies have demonstrated the mechanism of bone marrow suppression. ${ }^{7,8}$ This will be reflected in the peripheral blood as reduced counts of two or more than two cell lines.

Studies have also demonstrated that the Dengue virus have a tropism for hematopoietic cells and can proliferate in these cell lines without causing cytopathic effect. This enables them to sustain and propagate in this cell lines. ${ }^{8}$ Our study showed that less than half of the study subjects had indirect evidence of bone marrow suppression at the time of diagnosis.

The pattern of changes that occur in bone marrow is studied in experimental set up. This study has shown that the cell lines in the bone marrow get suppressed in the initial stage in viral infection and the nadir is seen on day 4 or 5 of clinical manifestation of fever. ${ }^{9}$ However, this suppressive effect can be seen up to 10 days after the cell lines get infected. ${ }^{10}$ These experimental findings have been reflected in the real life scenario as seen in our study. Maximum number of patients had the multiple cell line suppression during the $\operatorname{IgG}$ phase of the illness, which is typically seen about 10 days after infections. Second higher peak in the number of patients was seen in the early phase of clinical illness where only NS1 antigen was positive. This again supports the earlier finding that the bone marrow suppression starts about 4 to 5 days after infection which coincides with the onset of fever following incubation period.

Dengue virus does affect all the cell lines in the bone marrow with an increased tropism for erythrocytic line. ${ }^{8}$ Given this fact, it is reasonable to expect predominant decrease in the peripheral blood red cell counts. However, antibody response to the viral infection and an immunological cascade results in capillary leak $k^{5,11,12}$ and hence result

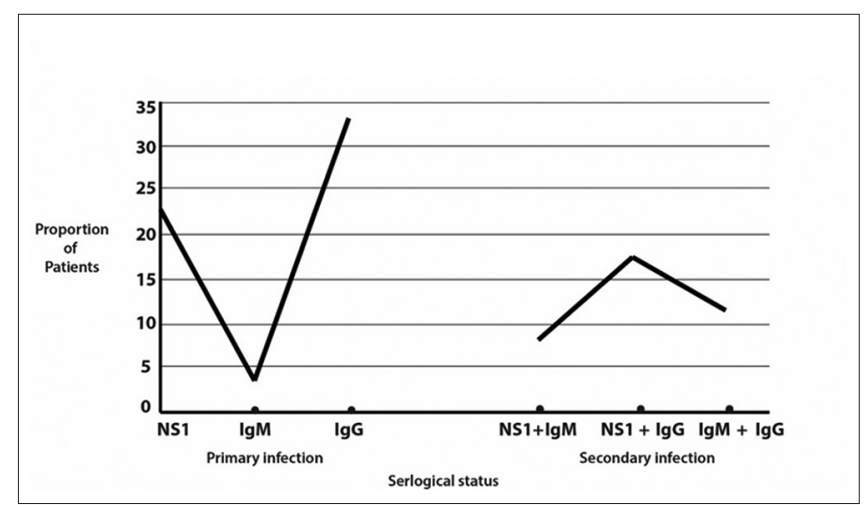

Figure 1: Proportion of patients with decreased peripheral cell line (2 or $>2$ ) in relation to various serological phases of dengue infection 
inhemoconcentration. This can spuriously elevate the peripheral blood red cell count. In our study we had $26.9 \%$ of our patients having haematocrit below 35\%. This could be explained by the fact that either patients were significantly anaemic prior to contracting this infection or there was bone marrow suppression as a predominant pathogenic mechanism and capillary leak was insignificant.

These patients could form a subset whose thrombocytopenia may theoretically respond to corticosteroids as demonstrated in few clinical studies. ${ }^{13,14}$ Conversely majority of the clinical studies have failed to demonstrate this benefit. ${ }^{15}$ Future studies segregating the patients with thrombocytopenia with different mechanisms and targeting the treatment may provide the answers to the dogma of steroid usage in dengue infection.

\section{CONCLUSION}

Nearly $40 \%$ of the patients will have decrease in multiple cell lines in the peripheral blood suggesting bone marrow suppression at the time of diagnosis of Dengue infection. Highest proportion $(33 \%)$ is seen during post primary phase where only $\operatorname{IgG}$ antibody will be positive followed by patients in the initial stage of the infection where NS1 antigen alone will be positive (23\%).

\section{REFERENCES}

1. WHO fact sheet on Dengue. Cited $26^{\text {th }}$ September 2015. Available from: URL: http://www.who.int/mediacentre/factsheets/ fs 117/en.

2. Sridharan A, Chen Q, Tang KF, Ooi EE, Hibberd ML and Chen J. Inhibition of megakaryocyte development in the bone marrow underlies dengue virus-induced thrombocytopenia in humanized mice. Journal of virology 2013;87(21):11648-11658.

3. Guabiraba R and Ryffel B. Dengue virus infection: current concepts in immune mechanisms and lessons from murine models. Immunology 2014;141(2):143-156.

4. Rothwell SW, Putnak R and La Russa VF. Dengue-2 virus infection of human bone marrow: characterization of dengue-2 antigen-positive stromal cells. American Journal of Tropical Medicine andHygiene 1996;54(5):503-510.

5. Saito M, OishiK, Inoue S, Dimaano EM, Alera MT, Robles AM, etal. Association of increased platelet-associated immunoglobulins with thrombocytopenia and the severity of disease in secondary dengue virus infections. Clinical \& Experimental Immunology 2004;138(2):299-303.

6. Noisakran S, Onlamoon N, Hsiao HM, Clark KB, Villinger F, Ansari AA, et al. Infection of bone marrow cells by Dengue virus in vivo. ExpHematol2012; 40:250-252.

7. Jennifer L, Kyle P, Robert B and Eva H. Dengue Virus Infects Macrophages and Dendritic Cells in a Mouse Model of Infection. The Journal of Infectious Diseases 2007; 195:1808-1817.

8. Shinji N, Ching JL and Neal SY. Dengue virus, A Flavivirus, Propagetes in Human Bone marrow progenitors and hematopoietic cell lines. Blood 1989; 74:1235-1240.

9. La Russa VF and Innis BL. Mechanisms of Dengue virusinduced bone marrow suppression. Baillieres Clin Haematol 1995; 8:249-270.

10. Sansanee $\mathrm{N}$, Nattawat $\mathrm{O}$, Hui-Mien $\mathrm{H}$, Kristina BC, Francois $\mathrm{V}$, Aftab A, et al. Infection of bone marrow cells by Dengue virus in vivo. ExpHematol 2012; 40:250-259.

11. Wahala WM and De Silva AM. The human antibody response to dengue virus infection. Viruses 2011;3(12):2374-2395.

12. Azeredo EL, Monteiro $\mathrm{RQ}$ and de-Oliveira Pinto LM. Thrombocytopenia in dengue: interrelationship between virus and the imbalance between coagulation and fibrinolysis and inflammatory mediators. Mediators of inflammation 2015; 2015:313842.

13. Verma SP, Hamide A, Wadhwa J and Sivamani K. Corticosteroid responsive prolonged thrombocytopenia in a case of dengue fever. BMJ Case Reports 2013; 2013:200249.

14. Shashidhara KC, Murthy KS, Gowdappa HB and Bhograj A. Effect of high dose of steroid on plateletcount in acute stage of dengue fever with thrombocytopenia. Journal of clinical diagnosis and research2013; 7:1397-1400.

15. Rajapakse S, Rodrigo C, Maduranga S and Rajapakse AC. Corticosteroids in the treatment of dengue shock syndrome. Infection and Drug Resistance2014; 7:137-143.

Authors Contribution:

VK - Concept, review of literature, manuscript preparation and critical review of final manuscript; RR - Review of literature, collection of data and manuscript preparation; SMD - Concept, study design, statistical analysis, manuscript preparation and its critical review.

Source of Support: Nil, Conflict of Interest: None declared. 